\title{
Applying Delaunay triangulation augmentation for deep learning facial expression generation and recognition $^{\star}$
}

\author{
Hristo Valev ${ }^{1,2}[0000-0001-8157-4097]$, Alessio Gallucci ${ }^{3[0000-0002-6758-8131]}$, Tim \\ Leufkens $^{1[0000-0002-2807-0532]}$, Joyce Westerink ${ }^{1,3[0000-0002-2391-0387]}$, and \\ Corina Sas 2 [0000-0001-9297-9612] \\ 1 Philips Research, High Tech Campus 34, 5656 AE Eindhoven, The Netherlands \\ 2 Lancaster University, Bailrigg, Lancaster LA1 4YW, United Kingdom \\ 3 Technical University Eindhoven, Groene Loper 3, 5612 AE Eindhoven, The
} Netherlands

\begin{abstract}
In this paper, we describe the use of Delaunay triangulation to blend images of faces, which allows us to create and automatically label facial expressions portraying varying intensities of emotion. We have applied this technique on the RafD dataset consisting of 67 participants and 8 categorical emotions and evaluated the augmentation in two ways - using a facial expression generation and recognition tasks with deep learning models. For the generation task, we used a deconvolution neural network which learns to encode the input images in a high-dimensional feature space and generate realistic expressions at varying intensities. The augmentation significantly improves the quality of images compared to previous comparable experiments and it allows to create images with a higher resolution. For the recognition task, we evaluated pre-trained Densenet121 and Resnet50 networks with either the original or augmented dataset. Our results indicate that the augmentation alone has a similar or better performance compared to the original. Implications of this method and its role in improving existing facial expression generation and recognition approaches are discussed.
\end{abstract}

Keywords: facial expressions, augmentation, deep learning, emotions

\footnotetext{
* This work has been supported by AffecTech: Personal Technologies for Affective Health, Innovative Training Network funded by the H2020 People Programme under Marie Skłodowska-Curie grant agreement No 722022.
} 


\section{Introduction}

Generating and recognizing facial expressions has numerous applications ranging from medical ones, such as inferring character [1], emotional states and intent [2], detection of diseases [3] to authentication and biometrics [4], designing affective interfaces [5-8] and virtual avatars [9, 10] and computer graphics [11]. State of the art uses machine- and deep-learning methods to achieve impressive results $[12,13]$. However, most approaches are able to recognize or synthesize facial expressions as a categorical state with few recent works being able to also account for varying emotion intensities $[14,15]$. This is important as facial expressions' meaning varies at different intensities and such approaches need to be able to distinguish the intensity portrayed emotion accurately [16].

While both facial expression recognition and generation approaches have achieved impressive results in recent years, humans are still better recognizing subtle facial expressions [17]. A reason for that is the scarce number of datasets consisting of labeled nuanced expressions. In fact, correctly classifying subtle facial expressions poses a few key challenges: 1) facial expressions displaying low intensity of emotion can share certain similarities with other expressions, 2) dissimilarity in the display of emotion between different people and 3) imbalanced sample distribution for expressions and subjects [18].

In this paper, we explore an existing method for blending faces applied on a facial expression dataset and evaluate it in a generation and recognition tasks. First, we elucidate on the steps required to create an augmented dataset, then present the results from both tasks and discuss the implications of this method for improving upon both facial expression generation and recognition challenges and outline further use-cases.

\section{Dataset}

In order to train the models, we are using the Radboud Faces Database (RafD) [19]. The dataset consists of 8040 colored images with $681 \times 1024$ resolution including 67 persons -57 adults and 10 children. The dataset is labeled for person's identity, gender, ethnicity, facial expression, eye gaze direction, and camera angle. For the purpose of simplifying the method and evaluation described herein, we only used images of front-facing facial expressions, omitting angles different than $90^{\circ}$. Furthermore, we are also only including images of adults which results in 1336 images.

\section{Augmented dataset}

In this section we outline the pre-processing steps required to create the augmented dataset. For the preprocessing steps of alignment, centering and cropping we used the python libraries dlib, imutils and OpenCV2. For computing the Delaunay triangulation and affine transform we used OpenCV2. 


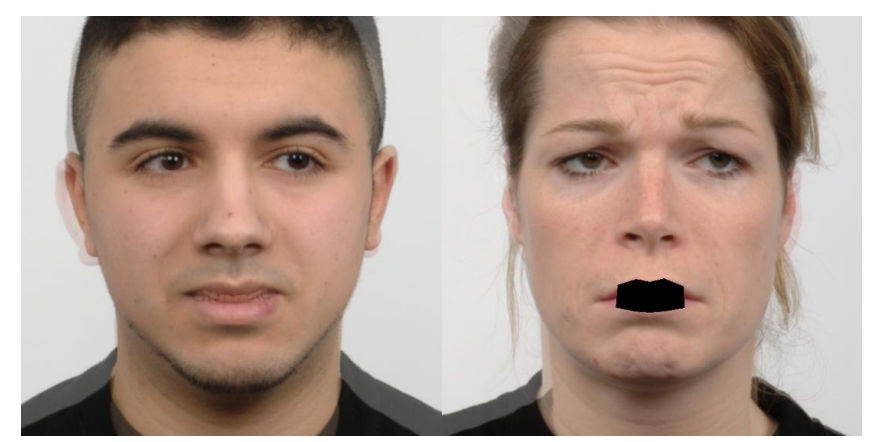

Fig. 1. On the left is a successful augmentation portraying happiness with 0.3 intensity. On the right is an unsuccessful augmentation.

\subsection{Alignment, Centering \& Cropping}

In the first phase, we detect and locale 68 landmarks with dlib. The landmarks are then used to align all the images with the FaceAligner class from imutils with rigid registration. Generally, the images in the RafD dataset are well aligned, however, there is still a benefit in using the aligner as we noticed a slight tilt in some of them. This has yielded some improvements, particularly for features such as the eyes and mouth, since it ensures that those are stacked on the same spatial coordinates in every image. Finally, detected faces are cropped out of the original image with output dimensions of 550x550 pixels.

\subsection{Computing Delaunay triangulation and Transform}

To generate the augmented dataset, we computed Delaunay triangulation on the aligned and centered images by re-identifying landmark points after the preprocessing steps described earlier using dlib. Computing the Delaunay trinagulation can be done in multiple ways [20]. We have relied on the OpenCV2 implementation as it is a freely available software and the results can be easily reproduced. The point-to-point correspondence registration is trivial, since the landmarks descriptive of facial features are an ordered set. Subsequently the rasterized images using the computed Delaunay trinagulation are morphed together, where corresponding landmark points are interpolated by a factor between $[0,1]$ weighing each image's contribution. Applying an affine transformation, we calculate each pixel's new position in the blend in order to overlap the rasterized textures from both images and output a morphed blended image. To create the augmented dataset, we have applied this method per expression and within subjects. For each augmentation, we took the neutral and a 'target' facial expression, where the result of the method portrays the latter's expression at half intensity. For example, an even blend between the neutral and happy expressions will produce a smiling facial expression with an intensity of 0.5 . The algorithm can also be applied to produce varying levels of intensities by adjusting the blending factor. 


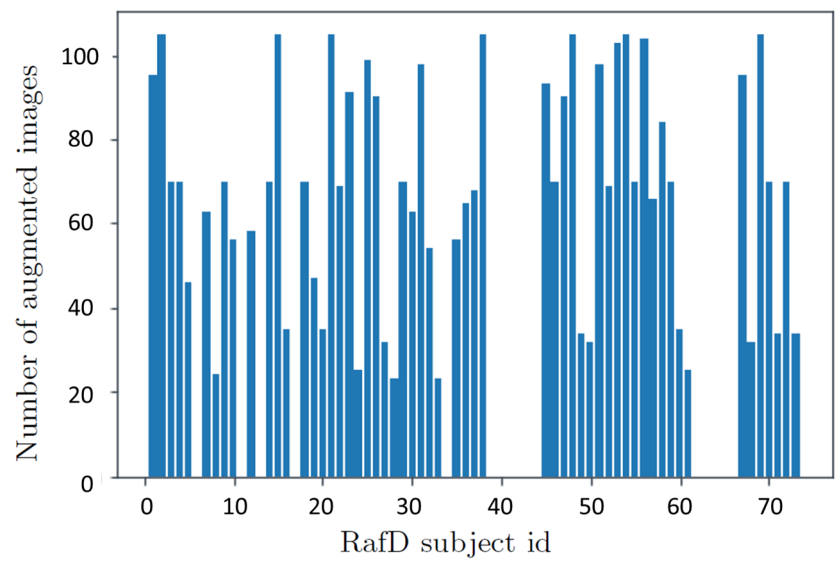

Fig. 2. Augmentations per subject (as referenced by their ids in the dataset) without artefacts. Optimally, we expect 105 images per subject for the [0.3, 0.7] intensity range descriptive of 5 intensities, 7 emotions and 3 eye gaze directions.

We created expression intensities between 0.3 and 0.7 at increments of 0.1 as expressions with no (neutral expression) or maximum intensity are already contained within the original dataset. By applying this augmentation per person and then per expression, while using the neutral expression as a baseline, our approach yielded 6840 images. In some cases, mostly for the neutral expression for particular subjects, the augmentation produces visual artefacts, which makes them unsuitable for use (see Figure 1). Figure 2 depicts the amount of augmented images created per subject. Noticeable is that there are clusters with near-similar amount of augmentations, due to the fact that the visual artefacts manifested on particular images, which rendered any blends thereof unusable. Excluding unsuccessful augmentations, the final augmented dataset consisted of 3848 images. Table 1 visualizes the distribution of created augmentations according to expression and intensity of displayed emotion. It consists of mostly evenly distributed number of augmentations for intensities. There are also less samples for sad, angry and contemptuous facial expressions in contrast to happy, disgusted, fearful and surprised ones.

\subsection{Limitations}

Visual artefacts expressed as solid black regions produced by the transformation were mostly localized in the area around the mouth, such that the subsequent interpolation between both sets of points fails (see Figure 1). In our approach we are blending expressions within subjects, this was observed in nearly half of the generated augmentations. Those images need to be discarded from the final dataset as they will likely severely hamper the performance in the subsequent 
Table 1. Augmented images without artefacts per step

\begin{tabular}{cccccc}
\hline \hline Emotion $\backslash$ Label & 0.3 & 0.4 & 0.5 & 0.6 & 0.7 \\
happy & 122 & 122 & 122 & 122 & 122 \\
sad & 98 & 98 & 100 & 99 & 98 \\
angry & 93 & 91 & 85 & 81 & 77 \\
contemptuous & 103 & 102 & 102 & 98 & 99 \\
disgusted & 122 & 122 & 122 & 122 & 122 \\
fearful & 119 & 118 & 119 & 119 & 119 \\
surprised & 122 & 122 & 122 & 122 & 122 \\
\hline total & 779 & 775 & 772 & 763 & 759 \\
\hline
\end{tabular}

generation and recognition tasks. Using this approach, we are able to align facial features together and create a blended image, however, regions, which lie outside the face contours are simply added and averaged together. Consequently, hairstyles of all blended subjects are visible in the resulting images (see Figure 1). This effect is marginal in this case, as subjects in the RafD mostly have the same hairstyle in their depictions of different facial expressions.

\section{Facial expression generation task}

The model we used in the generation task is a deconvolutional decoder network, previously used for morphing of objects [21]. The relative simplicity of the model allows to better control and interpret the output, in contrast to more sophisticated GANs. Training images are encoded in a high-dimensional latent space, whereby novel representations are created by varying the input parameters and iterating over it. The original model includes as an additional output a segmentation mask, which unnecessarily complicates the model and was removed. We implemented the model in tensorflow as it gives us more flexibility in fine-tuning individual layers and applying minor optimizations. For training, we used both the original RafD dataset and the augmented one. The model loss is computed based on the labels from the original dataset and the soft labels created by the augmentation. We used an early-stopping criterion, which concluded the training after 96 epochs.

Figure 3 displays the results from the generative model for the facial expressions of happiness, sadness, disgust, anger, fear, surprise and contempt each presented in successive rows. Images on the left depict expressions at their maximum intensity and each subsequent image to the right represents a decrement of 0.1 in portrayed emotion intensity towards the neutral expression.

The model was able to produce realistic-looking depictions of facial expressions for all emotion intensities with particular facial features appearing crisp and detailed. We discovered experimentally that using instance normalization produced significantly better results to batch normalization. Furthermore, uneven class distribution, as expected, plays a role when the amount of augmented training samples outweighs the original dataset resulting in the model learning 


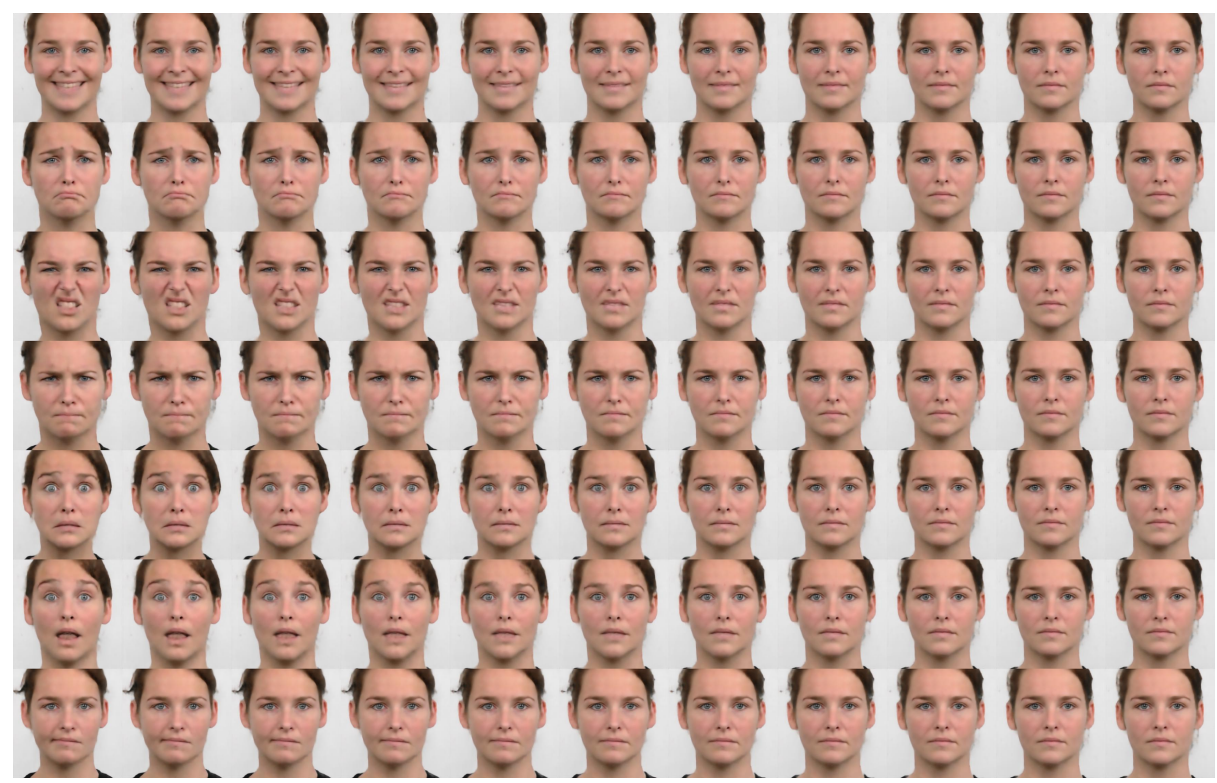

Fig. 3. Output from the generative model trained on the original and augmented datasets. Images on the right depict the neutral expression while those on the left - the facial expressions for happiness, sadness, disgust, anger, fear, surprise and contempt at maximum intensity. The intermediary images are samples in-between at increments of 0.1 .

artefacts from the augmentation. The number of samples between the augmented and original datasets is at a proportion of approximately $3: 1$. We, therefore, balanced the training set to a uniform distribution by oversampling the originals, which mediated the best results.

In contrast, faces generated only with the original RafD dataset appear blurred and the intermediary expressions are rather ambiguous. Omitting the augmentation does not provide sufficient training data for the generative model to learn textures such as those for teeth. In this case, the model substitutes textures for teeth for those of lips or skin in intermediary expressions for emotions such as anger and happiness. This hints at the observation that the network does not learn the meaning behind facial expressions or rather the interaction between individual features, albeit it learns low-level and in subsequent layers high-level representations in order to adequately generate them. Using the augmentation alone does not create perfect synthetic facial expressions either as it is prone to artefacts itself (i.e. shadows when blending images of people with different hair styles). Using the augmentation in combination with the original dataset provides suitable synthetic images for the model to learn accurate approximations for intermediary facial expressions. This is also the case for subjects in the dataset, for which there were relatively few augmentations available as previously elaborated on why. 


\section{Facial expression recognition task}

To establish whether the augmentation can improve results for facial expression recognition classification, we designed a downstream task evaluating the quality of the augmentation. In this manner we can compare the performance efficacy of networks trained on the original dataset and the augmentation. We used the DenseNet121 [22] and ResNet50 [23] networks as they have achieved impressive results in various classification tasks. A suitable metric for evaluating generative models is the Classification Accuracy Score (CAS) [24]. Both DenseNet121 and ResNet50 have been pre-trained on ImageNet [25]. For ground truth, we used the soft labels for the facial expression intensities as generated by the augmentation. It is important to note that, while the soft labels (e.g. intensities of emotions) in the original dataset are either 1 or 0 as there are no intermediary expressions, the augmented one only consists of ones in the intensity range $[0.3,0.7]$ as shown (see Table 1). For training, we split both original and augmented datasets in a $90 \%$ training- and $10 \%$ test-sets stratified according to subject. For testing, since we are evaluating the augmentation as a downstream task, we are only using the test-set from the original dataset, which as mentioned above includes only binary labels. We have also adapted the classification for the DenseNet121 and ResNet50 models to predict 7 classes, representing expressions available in the dataset, in place of the default 1000 classes. For training parameters we used ADAM optimizer [26] with $\beta_{1}=0.9, \beta_{2}=0.99, \epsilon=10^{-8}$, learning rate $\alpha=0.001$ and no weight decay. We also evaluated an additional augmentation strategy where in A) we are not using any augmentation and in B) we are further using standard augmentations consisting of rotation $(+-30)$, scaling with a random factor between (0.8 and, 1.2) and shear random factor (0.9 and, 1.1). The results of our experiment can be seen in Table 2 .

Table 2. Facial expression recognition results from Resnet50 and Densenet121 models where Augmented and Original refer to the RafD dataset and A) is no additional augmentation and B) is applying rotation (+-30), scaling with a random factor between (0.8 and, 1.2) and shear random factor (0.9 and, 1.1).

\begin{tabular}{ccccc}
\hline \hline & Densenet121 & \multicolumn{3}{c}{ Resnet50 } \\
& Augmented & Original & Augmented & Original \\
A & 0.965 & 0.965 & 0.972 & 0.965 \\
B & 0.993 & 0.958 & 0.958 & 0.972 \\
\hline
\end{tabular}

While the performance for the RafD dataset are reaching human level of performance (recognizing simple emotions in high resolution images is a relatively simple task for deep learning models), the results hold true also for the augmented data. In addition, using only the augmented images performs better, or on pair, compared to the original images in every situation except for Resnet50 B). An interesting observation is that augmented images do not include facial expressions at maximum intensity but have been evaluated on such. 
This implies that training on augmented images featuring medium intensities of portrayed emotions was sufficient to classify those at maximum intensity as well. In our case, cross-entropy with soft labels is not symmetric due to the fact that 1) soft-labels in the augmentation weigh towards the neutral expression and 2) the augmentation for certain expressions is more prone to artefacts (i.e. sadness and anger), which results in a unbalanced training samples between classes. Contrary to our belief, however, this did not appear to hamper the CAS scores for the model trained only on augmented images. This is a positive result, as it implies that the networks learn class distribution on- or almost on-par and in some cases even better compared to using the original dataset, which makes both datasets practically interchangeable for training.

\section{Discussion}

In this paper we have presented a known approach for blending images of faces and explored its use to augment existing facial expression datasets to create labeled expressions portraying varying intensities of emotion. We evaluated the augmentation in a facial expression generation and recognition tasks and our results indicate significant improvements in generating facial expressions when using the augmentation in addition to the original dataset. In a facial expression recognition task, the original and augmented datasets can be used interchangeably as both achieve near-similar levels of classification accuracy. There are benefits to using this augmentation for a wider set of tasks, in particular for networks which also aim to recognize intensities of facial expression beyond categorical classification.

Some further considerations when using this technique are that the method can also be applied in a person-agnostic manner as long as facial feature landmarks can be correctly identified. For the purposes of our evaluation, we applied the augmentation only within subjects, but this can be done for an arbitrary amount of identities. Those blended pseudo-identities would still be of sufficient quality to be reliably used. A potential benefit is that with an increase of blended identities, artefacts caused by the augmentation method are reduced, which in turn allows the generation of larger automatically labeled datasets. Recently, it has also been a topic of discussion that a lot of datasets are biased and are not representative for group diversity. This method can be particularly useful to correct for this bias by generating morphed samples for underrepresented genders and ethnicities which in turn can be helpful in producing less biased algorithms.

Holistic-based facial-expression recognition approaches can benefit from such augmentation as well. It can provide plausible samples for training, which can help discriminative models to learn more accurate decision boundaries between classes, thus reducing misclassification errors for nuanced facial expressions. Currently, more expressive features of the face are weighted such that they disproportionately influence classification. Alternatively, the presented method can be used in recognition tasks to strengthen the interdependence of formant facial features contributing to individual facial expressions. 


\section{Future work}

In further work, we aim to evaluate this approach on datasets, ideally containing ambiguous and nuanced expressions. We also aim to evaluate the effectiveness of this method in more challenging tasks, such as images with low-resolution, under different lighting conditions or with occlusions. In addition, we would also like to conflate both generation and recognition experiments in a downstream task, evaluating whether a combination of the generative model output trained on augmented data can further improve the augmentation quality. In turn, this can be used to also improve the performance of facial expression recognition tasks. Finally, we would also like to investigate whether training a classification model with augmented data for intermediary intensities can also improve recognition results for nuanced expressions.

\section{Conclusion}

We have evaluated an effective and inexpensive method to create a labeled augmented facial expression dataset with varying intensities of emotion from a categorically labeled one. We have detailed the steps required for its creation and explored potential caveats when working with the augmented data such as uneven sample distribution of labeled classes, particularly when working with soft labels. The simplicity of the method makes it easily applicable for facial expression recognition and generation tasks. The augmentation relying on a geometric transformation makes experiments using this approach easily reproducible. The application of this augmentation can address contemporary problems in deep learning algorithms related to improvements in nuanced facial expression recognition and generation, uneven sample distribution and debiasing facial expression datasets. 


\section{References}

1. E. J. Cogsdill, A. T. Todorov, E. S. Spelke, and M. R. Banaji, "Inferring character from faces: A developmental study," Psychological science, vol. 25, no. 5, pp. 11321139, 2014.

2. R. Buck, "Social and emotional functions in facial expression and communication: The readout hypothesis," Biological psychology, vol. 38, no. 2-3, pp. 95-115, 1994.

3. P. Kruszka, Y. A. Addissie, D. E. McGinn, A. R. Porras, E. Biggs, M. Share, T. B. Crowley, B. H. Chung, G. T. Mok, C. C. Mak et al., "22q11. 2 deletion syndrome in diverse populations," American Journal of Medical Genetics Part A, vol. 173, no. 4, pp. 879-888, 2017.

4. K. I. Chang, K. W. Bowyer, and P. J. Flynn, "Multiple nose region matching for 3d face recognition under varying facial expression," IEEE Transactions on Pattern Analysis and Machine Intelligence, vol. 28, no. 10, pp. 1695-1700, 2006.

5. H. Valev, T. Leufkens, C. Sas, J. Westerink, and R. Dotsch, "Evaluation of a self-report system for assessing mood using facial expressions," in International Symposium on Pervasive Computing Paradigms for Mental Health. Springer, 2019, pp. 231-241.

6. P. Sanches, A. Janson, P. Karpashevich, C. Nadal, C. Qu, C. Daudén Roquet, M. Umair, C. Windlin, G. Doherty, K. Höök et al., "Hci and affective health: Taking stock of a decade of studies and charting future research directions," in Proceedings of the 2019 CHI Conference on Human Factors in Computing Systems, 2019, pp. 1-17.

7. D. Colombo, J. Fernández-Álvarez, C. Suso-Ribera, P. Cipresso, H. Valev, T. Leufkens, C. Sas, A. Garcia-Palacios, G. Riva, and C. Botella, "The need for change: Understanding emotion regulation antecedents and consequences using ecological momentary assessment." Emotion, vol. 20, no. 1, p. 30, 2020.

8. M. Alfaras, V. Tsaknaki, P. Sanches, C. Windlin, M. Umair, C. Sas, and K. Höök, "From biodata to somadata," in Proceedings of the 2020 CHI Conference on Human Factors in Computing Systems, 2020, pp. 1-14.

9. L. Hu, S. Saito, L. Wei, K. Nagano, J. Seo, J. Fursund, I. Sadeghi, C. Sun, Y.-C. Chen, and H. Li, "Avatar digitization from a single image for real-time rendering," ACM Transactions on Graphics (ToG), vol. 36, no. 6, pp. 1-14, 2017.

10. A. Gallucci, D. Znamenskiy, and M. Petkovic, "Prediction of 3d body parts from face shape and anthropometric measurements," Journal of Image and Graphics, vol. 8, no. 3, 2020.

11. S. Lombardi, J. Saragih, T. Simon, and Y. Sheikh, "Deep appearance models for face rendering," ACM Transactions on Graphics (TOG), vol. 37, no. 4, pp. 1-13, 2018.

12. C. Chu, A. Zhmoginov, and M. Sandler, "Cyclegan, a master of steganography," arXiv preprint arXiv:1712.02950, 2017.

13. Y. Choi, M. Choi, M. Kim, J. W. Ha, S. Kim, and J. Choo, "StarGAN: Unified Generative Adversarial Networks for Multi-domain Image-to-Image Translation," Proceedings of the IEEE Computer Society Conference on Computer Vision and Pattern Recognition, pp. 8789-8797, 2018.

14. H. Ding, K. Sricharan, and R. Chellappa, "Exprgan: Facial expression editing with controllable expression intensity," arXiv preprint arXiv:1709.03842, 2017.

15. A. Pumarola, A. Agudo, A. M. Martinez, A. Sanfeliu, and F. Moreno-Noguer, "Ganimation: Anatomically-aware facial animation from a single image," in Proceedings of the European conference on computer vision (ECCV), 2018, pp. 818833. 
16. M. Rychlowska, R. E. Jack, O. G. Garrod, P. G. Schyns, J. D. Martin, and P. M. Niedenthal, "Functional smiles: Tools for love, sympathy, and war," Psychological science, vol. 28, no. 9, pp. 1259-1270, 2017.

17. N. Yitzhak, N. Giladi, T. Gurevich, D. S. Messinger, E. B. Prince, K. Martin, and H. Aviezer, "Gently does it: Humans outperform a software classifier in recognizing subtle, nonstereotypical facial expressions." Emotion, vol. 17, no. 8, p. 1187, 2017.

18. A. C. Le Ngo, R. C.-W. Phan, and J. See, "Spontaneous subtle expression recognition: Imbalanced databases and solutions," in Asian conference on computer vision. Springer, 2014, pp. 33-48.

19. O. Langner, R. Dotsch, G. Bijlstra, D. H. Wigboldus, S. T. Hawk, and A. Van Knippenberg, "Presentation and validation of the radboud faces database," Cognition and emotion, vol. 24, no. 8, pp. 1377-1388, 2010.

20. D.-T. Lee and B. J. Schachter, "Two algorithms for constructing a delaunay triangulation," International Journal of Computer $\&$ Information Sciences, vol. 9, no. 3, pp. 219-242, 1980.

21. A. Dosovitskiy, J. T. Springenberg, M. Tatarchenko, and T. Brox, "Learning to generate chairs, tables and cars with convolutional networks," IEEE transactions on pattern analysis and machine intelligence, vol. 39, no. 4, pp. 692-705, 2016.

22. G. Huang, Z. Liu, L. Van Der Maaten, and K. Q. Weinberger, "Densely connected convolutional networks," in Proceedings of the IEEE conference on computer vision and pattern recognition, 2017, pp. 4700-4708.

23. K. He, X. Zhang, S. Ren, and J. Sun, "Deep residual learning for image recognition," in Proceedings of the IEEE conference on computer vision and pattern recognition, 2016, pp. 770-778.

24. S. Ravuri and O. Vinyals, "Classification accuracy score for conditional generative models," in Advances in Neural Information Processing Systems, 2019, pp. 12268 12279 .

25. O. Russakovsky, J. Deng, H. Su, J. Krause, S. Satheesh, S. Ma, Z. Huang, A. Karpathy, A. Khosla, M. Bernstein et al., "Imagenet large scale visual recognition challenge," International journal of computer vision, vol. 115, no. 3, pp. 211-252, 2015.

26. D. P. Kingma and J. Ba, "Adam: A method for stochastic optimization," arXiv preprint arXiv:1412.6980, 2014. 